\title{
Evaluating the Student-Teacher Relationship Scale in Italian Young Children: An Exploratory Structural Equation Modeling Approach
}

\author{
Stefania Sette', Antonio Zuffianòㄹ , Fabio Lucidi', \\ Fiorenzo Laghi', Antonia Lonigro', and Emma Baumgartner'
}

\begin{abstract}
The study analyzed the factorial and concurrent validity of the Student-Teacher Relationship Scale (STRS) using an exploratory structural equation modeling (ESEM) approach. Participants were 368 Italian children aged 3 to $6(M=4.60, S D=0.98)$. The three-factor ESEM solution fit the data better than the classical confirmatory factor analysis (CFA) model and the measurement invariance of the scale was confirmed across sex and age (3-4 vs. 5-6 years) groups. The concurrent validity of the STRS was investigated within the ESEM approach using children's social behaviors as validity criteria. Findings supported the goodness of ESEM over CFA and attested to the validity of the STRS to understanding the teacher-child relationship quality in young children.
\end{abstract}

\section{Keywords}

teacher-child relationships, STRS, ESEM, measurement invariance, young children

In the preschool and kindergarten educational context, the quality of teacher-child relationships plays an important role in explaining children's social behavior within the classroom (Hughes, Bullock, \& Coplan, 2014). Whereas secure or close teacher-child relationships have been associated with higher levels of socially competent behaviors, school liking, and peer acceptance, negative or insecure teacher-child relationships have been related to children's behavioral difficulties (e.g., aggressive or withdrawn behaviors; Sabol \& Pianta, 2012).

Given the importance of such relationships, developmental scientists have pointed out the need for developing valid and reliable tools aimed at assessing the quality of teacher-child relationships in the earliest stages of childhood development. The Student-Teacher Relationship Scale (STRS; Pianta, 2001), a 28-item scale, is considered one of the most popular teacher-report tools to evaluate three core dimensions of relationships between teachers and children (i.e., close, conflictive, and dependent), as attested by its large use across different countries/cultures (e.g., Zhang \& Nurmi, 2012).

\footnotetext{
ISapienza University of Rome, Italy

2Liverpool Hope University, UK

Corresponding Author:

Stefania Sette, Via dei Marsi, 78, 00185, Rome, Italy.

Email address: stefania.sette@uniromal.it
} 
Despite the popularity of the STRS, previous studies based on exploratory factor analyses (EFA) and independent cluster models of confirmatory factor analyses (ICM-CFA) reported mixed findings about the factor structure of the STRS. For instance, Gregoriadis and Tsigilis (2008), using an EFA, found that a 26-item solution better captured the three-factor structure of the STRS. Milatz, Gluer, Harwardt-Heinecke, Kappler, and Ahnert (2014), using an ICM-CFA, dropped 16 items with poor psychometric properties to obtain a good factor solution. In addition, to the best of our knowledge, only a few studies evaluated the measurement invariance (MI) of the STRS across groups. For example, Milatz et al. (2014) supported a partial scalar invariance of the modified version of the STRS across kindergarteners, first graders, and second graders and a full scalar invariance of the scale across sex. Solheim, Berg-Nielsen, and Wichstrøm (2011) did not find full sex invariance on a modified 25-item version of the scale.

Based on these premises, the aim of the present study was to investigate the latent structure and concurrent validity of the STRS scores using an exploratory structural equation modeling (ESEM) framework, which is a flexible analytical technique that overcomes many limitations of EFA and ICM-CFA. Compared with highly restrictive basic ICM-CFA models that posit all cross-loadings on non-targeted factors to be zero, ESEM allows the free estimation of crossloadings (such as in classical EFA) while still offering the possibility of conducting MI tests such as in ICM-CFA. Furthermore, ESEM estimates less inflated correlations among latent factors (a problem which typically occurs in ICM-CFA; Morin, Marsh, \& Nagengast, 2015).

First, we expected a better fit of the ESEM model over the highly restrictive ICM-CFA. Second, we tested the MI of the STRS across age groups (preschoolers vs. kindergarteners) and sex. Finally, we conducted a concurrent validity analysis by examining the association of the STRS with children's socially competent, aggressive, and withdrawn behaviors (see Hughes et al., 2014; Sabol \& Pianta, 2012).

All these research goals were explored within the Italian preschool/kindergarten where children typically interact with same teachers and classmates for several years (Sette, Baumgartner, \& Schneider, 2014).

\section{Method}

\section{Participants}

Participants were 368 Italian children (49.7\% girls) between the ages of 3 and $6(M=4.60, S D=$ $0.98)$, enrolled in public preschools $(n=30$ classrooms). Written parental consent was obtained for all participants.

\section{Procedures}

To ensure the independence of measures, two teachers were used as sources of information in each classroom: One for the assessment of the teacher-child relationship quality (Teacher A) and the other for the evaluation of children's social behaviors (Teacher B).

\section{Measures}

The quality of the teacher-child relationship (Teacher A). The STRS (Pianta, 2001) was used to assess teachers' perceptions of their relationship with each child. The STRS contains 28 items rated on a 5-point Likert-type scale $(1=$ definitely does not apply to $5=$ definitely applies $)$ designed to assess closeness (11 items), conflict (12 items), and dependency (5 items).

Children's social behaviors (Teacher B). A revised version (Sette, Baumgartner, \& MacKinnon, 2015) of the Social Competence and Behavior Evaluation Scale (SCBE-30; LaFreniere \& Dumas, 
1996) was used to evaluate children's anxiety-withdrawal $(\alpha=.88)$, social competence $(\alpha=.89)$, and anger-aggression $(\alpha=.85)$.

\section{Data Analysis}

Given the ordered categorical nature of STRS items, we used the weighted least-squares mean and variance-adjusted (WLSMV) estimation method in Mplus 7.4 (Muthén \& Muthén, 2012). In addition, we took into account the hierarchical structure of our data (students nested within classrooms) by using the Mplus correction of standard errors for nested data (type = complex). Because the $\chi^{2}$ statistic is sensitive to sample size, we also used the comparative fit index (CFI) and Tucker-Lewis index (TLI) $>.90$, and root mean square error of approximation (RMSEA) values $<.08$ with $90 \%$ confidence interval $(\mathrm{CI})$ as indicative of acceptable model fit (Kline, 2011). To test differences among configural, metric, and scalar MI, we calculated $\Delta \chi^{2}$ tests. However, because the $\Delta \chi^{2}$ test is overly sensitive to minor model misspecifications, we also considered the $\Delta$ CFI test with a critical level of -.01 (Cheung \& Rensvold, 2002).

\section{Results}

The ICM-CFA model showed a worse fit, $\chi^{2}(347)=740.43, p<.001$, CFI $=.84$; TLI $=.83$, RMSEA $=.06,90 \% \mathrm{CI}=[.05, .06]$, compared with the ESEM with target rotation, $\chi^{2}(297)=$ $444.15, p<.001 ; \mathrm{CFI}=.94 ; \mathrm{TLI}=.93, \mathrm{RMSEA}=.04,90 \% \mathrm{CI}=[.03, .05]$. However, the ESEM indicated that six items presented very poor psychometric properties such as very low primary factor loadings ( $\lambda<.40$ on the intended factor; Schaefer et al., 2015) or a small gap between the primary and secondary loading (i.e., .20; Schaefer et al., 2015). Accordingly, we deleted these six items and we re-ran the analyses on the reduced 22-item version of the STRS. The ESEM confirmed its better fit, $\chi^{2}(168)=272.56, p<.001, \mathrm{CFI}=.96$; TLI $=.94, \mathrm{RMSEA}=.04,90 \% \mathrm{CI}=$ $[.03, .05]$, compared with the ICM-CFA model, $\chi^{2}(206)=404.45, p<.001$, CFI $=.92$; TLI $=.90$, RMSEA $=.05,90 \% \mathrm{CI}=[.05, .06]$. The statistically significant correlations at the latent level between (a) conflict-closeness and (b) conflict-dependency were less inflated in the ESEM ( $r \mathrm{~s}=$ -.39 and $.33, p s<.001$, respectively) compared with those estimated in the ICM-CFA ( $r \mathrm{~s}=-.49$ and $.54, p \mathrm{~s}<.001$, respectively), thereby suggesting the higher discriminant validity of the ESEM over the ICM-CFA (Morin et al., 2015). Importantly, although all items strongly loaded onto their intended factor, we also found statistically significant cross-loadings which attested to the unlikely goodness of the ICM-CFA model (see Table 1). Alpha reliability coefficients were .86, .88, and .63 for closeness, conflict, and dependency, respectively. The STRS also showed full scalar MI, thereby attesting to its strong factor consistency both across age groups and sex (see Table 2).

The ESEM used to test the validity of the STRS (Figure 1) fit the data relatively well, $\chi^{2}(263)=$ $368.64, p<.001, \mathrm{CFI}=.95$; TLI $=.93, \mathrm{RMSEA}=.03,90 \% \mathrm{CI}=[.03, .04]$, and explained a moderate amount of variance of children's anxiety-withdrawn $\left(R^{2}=.17\right)$, anger-aggression $\left(R^{2}=.24\right)$, and social competence $\left(R^{2}=.28\right)$. Younger children showed a higher tendency to engage in dependent teacher-child relationships $(\beta=-.21, p<.05)$. Girls $(0=$ boys, $1=$ girls $)$ were less likely than boys to have conflictive relationships with their teachers $(\beta=-.19, p<.05)$, whereas they tended to establish closer teacher-child relationships than boys $(\beta=.14, p<.05)$.

\section{Discussion}

To summarize, the ESEM approach corroborated the goodness of fit of the three-factor structure of the STRS in which closeness, conflict, and dependency are three distinct but interrelated facets of the teacher-child relationship quality. However, six out of 28 items of the scale proposed by Pianta (2001) were removed because they showed poor psychometric properties and were ambiguous in their content. For instance, the item related to the child's use of crying to get something from his or her teacher 
Table I. ESEM Standardized Factor Loadings for the Best Fitting Model.

\begin{tabular}{|c|c|c|c|c|}
\hline \multicolumn{2}{|c|}{ Abbreviated item content } & \multirow{2}{*}{$\frac{\text { Close TCR }}{.70^{* * * *}}$} & \multirow{2}{*}{$\frac{\text { Conflictive TCR }}{-.04}$} & \multirow{2}{*}{$\frac{\text { Dependent TCR }}{.23^{* * *}}$} \\
\hline $\mathrm{I}$. & affectionate, warm relationship & & & \\
\hline 3. & c. seeks comfort & $.69 * * *$ & .07 & $.35 * * *$ \\
\hline 5. & c. values our relationship & $.69 * * *$ & $-.20 * * *$ & $.26 * * *$ \\
\hline 7. & if praise, beams with pride & $.69 * * *$ & $-.13^{*}$ & .08 \\
\hline 9. & c. shares information & $.79 * * * *$ & .08 & $-.29 * * * *$ \\
\hline 12. & c. tries to please me & $.48 * * *$ & -.01 & $.14^{* *}$ \\
\hline 15. & easy to be in tune & $.71 * * *$ & $-.15^{* *}$ & $-.10 * *$ \\
\hline 27. & c. shares feelings and experiences & $.90 * * *$ & $.16 * * *$ & $-.33^{* * * *}$ \\
\hline 28. & $\begin{array}{l}\text { interactions with this child make me feel } \\
\text { confident }\end{array}$ & $.78 * * *$ & -.06 & -.05 \\
\hline 2. & struggling with each other & -.10 & $.71 * * *$ & -.04 \\
\hline 11. & c. is easily angry with me & $.15^{* *}$ & $.85^{* * * *}$ & .01 \\
\hline 13. & c. feels to be treated unfairly & $.09 *$ & $.8 I^{* * *}$ & -.08 \\
\hline 16. & c. perceives me as source of punishment & -.01 & $.83 * * *$ & -.13 \\
\hline 18. & c. remains angry or resistant & -.02 & $.78 * * *$ & -.08 \\
\hline 20. & c. drains my energy & -.09 & $.77^{* * *}$ & .05 \\
\hline 22. & difficult day when the $c$. is in a bad mood & $.11 *$ & $.82 * * *$ & $.14 * *$ \\
\hline 23. & c.'s feelings can be unpredictable & .01 & $.73 * * *$ & $.21 * *$ \\
\hline 24. & uncomfortable how c. and I get along & $-.29 * * *$ & $.54 * * *$ & $.14 *$ \\
\hline 26. & c. is manipulative with me & $-.23 * * *$ & $.54 * * *$ & $.11 *$ \\
\hline 8. & c. reacts strongly to separation & .10 & $.23 * * *$ & $.60 * * *$ \\
\hline 10. & c. is overly dependent on me & -.09 & .05 & $.81 * * * *$ \\
\hline 14. & c. asks help when there is no need & $.16 * *$ & $.24 * * *$ & $.5 I^{* * * *}$ \\
\hline
\end{tabular}

Note. Primary factor loadings are in bold. The six items removed were as follows: 4 . c. is uncomfortable with touch from me-reverse-scored item; 2I. c. copies my behavior (Close TCR); 19. c. responds to my look-reverse-scored item; 25. c. cries to get something from me (Conflictive TCR); 6. hurt, embarrassed when I correct; I7. c. expresses hurt or jealousy (Dependent TCR). ESEM = exploratory structural equation modeling; TCR = Teacher-child relationship; $c .=$ child.

$*_{p}<.05 . * * p<.01 . * * * p<.001$.

is not prototypical of the hypothesized dimension of conflict. Children at this age may cry when they want something, regardless of the quality of their relationship with teachers (secure vs. insecure). Interestingly, the removal of these items did not affect the content representation of each subscale and was consistent with previous studies on the factorial validity of the STRS (e.g., Milatz et al., 2014). Future studies are needed to replicate our findings in different cultural/educational contexts.

Importantly, the ESEM showed a better fit compared with the ICM-CFA. This is likely due to the fallacy of the items to capture a specific latent construct only as empirically attested by the significant cross-loadings of many items. The ESEM approach also confirmed its appropriateness to better differentiate the latent structure of STRS. Indeed, the associations among the three factors of the STRS were less inflated in the ESEM compared with the ICM-CFA model. We found that children who established secure relationships with their teachers were moderately less at risk to develop oppositional/conflictive relationships with them. In contrast, children with dependent relationships with teachers also displayed conflictive relationships with them (Sabol \& Pianta, 2012). However, future studies should replicate our results by using both ICM-CFA and ESEM methodologies.

The ESEM results also attested to the validity of the STRS in relation to children's social behaviors. Conflictive teacher-child relationships were related to lower levels of children's 


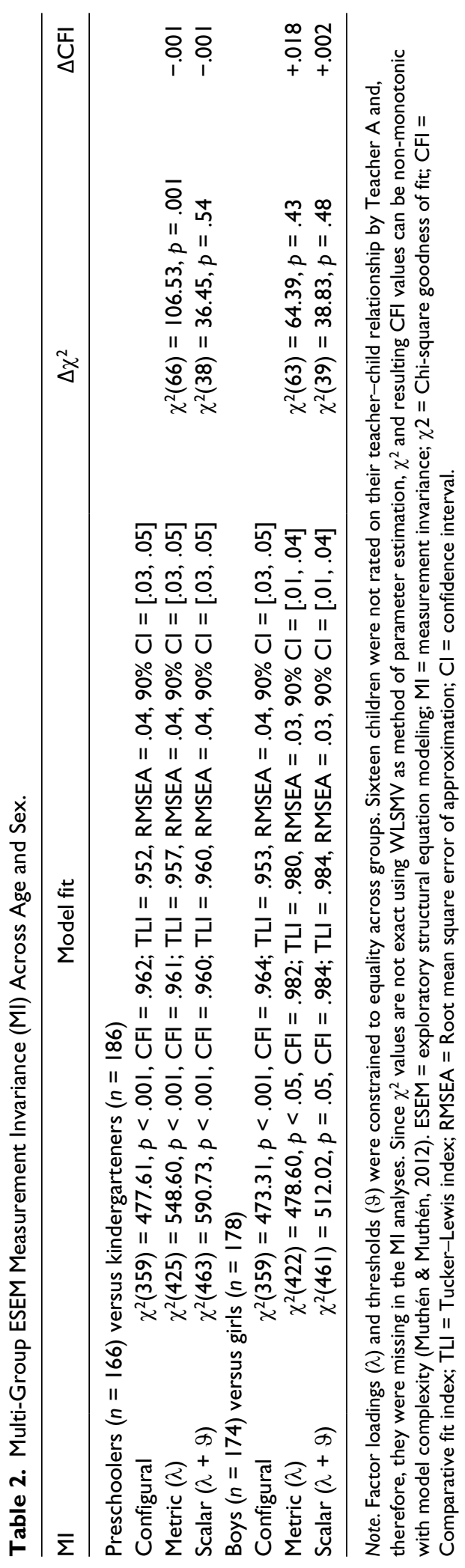




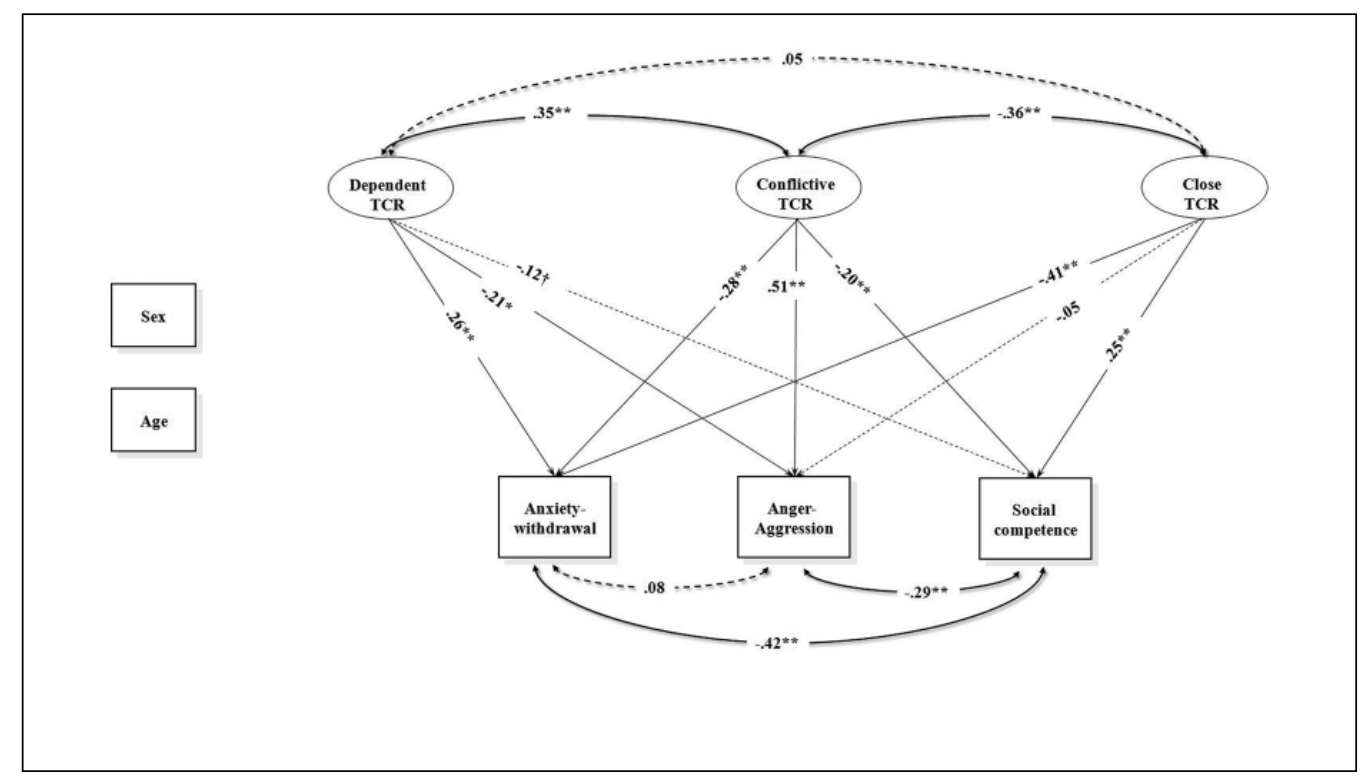

Figure I. ESEM validity model.

Note. Standardized coefficients were reported. For simplicity, the effects of sex and age, as well as factor loadings were estimated but not depicted. Dotted lines represent not significant paths $(p>.05)$. ESEM = exploratory structural equation modeling; TCR $=$ Teacher-child relationship. $t_{p}<.10 . * p<.05 . * * p<.01$.

anxious-withdrawn behaviors and social competence and higher levels of aggressive behaviors. This result is in line with previous studies which reported that conflictive teacher-child relationships were strong predictors of children's oppositional behaviors toward peers (Doumen et al., 2008). Furthermore, our results confirmed that dependent teacher-child relationships may enhance the risk of anxiety and withdrawn behaviors (Sabol \& Pianta, 2012). Hence, dependent children who have difficulties separating from their teachers may avoid interactions with peers during group activities or may have difficulties exploring independently the school environment.

In conclusion, the STRS represents a valid and reliable scale to help teachers understand the quality of their relationships with children. Italian teachers should be aware that supportive relationships with children from their earliest school experiences may prevent the onset of children's social difficulties (Pallini \& Laghi, 2012).

\section{Declaration of Conflicting Interests}

The author(s) declared no potential conflicts of interest with respect to the research, authorship, and/or publication of this article.

\section{Funding}

The author(s) received no financial support for the research, authorship, and/or publication of this article.

\section{References}

Cheung, G. W., \& Rensvold, R. B. (2002). Evaluating goodness of fit indexes for testing measurement invariance. Structural Equation Modeling: A Multidisciplinary Journal, 9, 233-255. doi:10.1207/ S15328007SEM0902_5 
Doumen, S., Verschueren, K., Buyse, E., Germeijs, V., Luyckx, K., \& Soenens, B. (2008). Reciprocal relations between teacher-child conflict and aggressive behavior in kindergarten: A threewave longitudinal study. Journal of Clinical Child \& Adolescent Psychology, 37, 588-599. doi:10.1080/15374410802148079

Gregoriadis, A., \& Tsigilis, N. (2008). Applicability of the Student-Teacher Relationship Scale (STRS) in the Greek educational setting. Journal of Psychoeducational Assessment, 26, 108-120. doi: $10.1177 / 0734282907306894$

Hughes, K., Bullock, A., \& Coplan, R. J. (2014). A person-centered analysis of teacher-child relationships in early childhood. British Journal of Educational Psychology, 84, 253-267. doi:10.1111/bjep.12029

Kline, R. B. (2011). Principles and practice of structural equation modeling (3rd ed.). New York, NY: Guilford Press.

LaFreniere, P. J., \& Dumas, J. E. (1996). Social Competence and Behavior Evaluation in children ages 3 to 6 years: The short form (SCBE-30). Psychological Assessment, 8, 369-377. doi:10.1037/10403590.8.4.369

Milatz, A., Gluer, M., Harwardt-Heinecke, E., Kappler, G., \& Ahnert, L. (2014). The Student-Teacher Relationship Scale revisited: Testing factorial structure, measurement invariance and validity criteria in German-speaking samples. Early Childhood Research Quarterly, 29, 357-368. doi:10.1016/j. ecresq.2014.04.003

Morin, A. J. S., Marsh, H. W., \& Nagengast, B. (2015). Exploratory structural equation modeling. In G. R. Hancock \& R. O. Mueller (Eds.), Structural equation modeling: A second course (2nd ed., pp. 395436). Charlotte, NC: Information Age.

Muthén, L. K., \& Muthén, B. O. (2012). MPlus user's guide: Statistical analysis with latent variables (Version 7). Los Angeles, CA: Author.

Pallini, S., \& Laghi, F. (2012). Attention and attachment related behavior toward professional caregivers in child care centers: A new measure for toddlers. The Journal of Genetic Psychology, 173, 158-174. doi:10.1080/00221325.2011.584330

Pianta, R. C. (2001). Student-Teacher Relationship Scale: Professional manual. Odessa, FL: Psychological Assessment Resources.

Sabol, T. J., \& Pianta, R. C. (2012). Recent trends in research on teacher-child relationships. Attachment \& Human Development, 14, 213-231. doi:10.1080/14616734.2012.672262

Schaefer, L. M., Burke, N. L., Thompson, J. K., Dedrick, R. F., Heinberg, L. J., Calogero, R. M., . . . Swami, V. (2015). Development and validation of the Sociocultural Attitudes Towards Appearance Questionnaire-4 (SATAQ-4). Psychological Assessment, 27, 54-67. doi:10.1037/a0037917

Sette, S., Baumgartner, E., \& Schneider, B. (2014). Shyness, child-teacher relationships, and socio-emotional adjustment in a sample of Italian preschool-aged children. Infant and Child Development, 23, 323-332. doi:10.1002/icd.1859

Sette, S., Baumgartner, E., \& MacKinnon, D. P. (2015). Assessing social competence and behavior problems in a sample of Italian preschoolers using the Social Competence and Behavior Evaluation scale (SCBE). Early Education and Development, 26, 46-65. doi:10.1080/10409289.2014.941259

Solheim, E., Berg-Nielsen, T. S., \& Wichstrøm, L. (2011). The three dimension of the Student-Teacher Relationship Scale: CFA validation in a preschool sample. Journal of Psychoeducational Assessment, 30, 250-263. doi:10.1177/0734282911423356

Zhang, X., \& Nurmi, J. (2012). Teacher-child relationships and social competence: A two-year longitudinal study of Chinese preschoolers. Journal of Applied Developmental Psychology, 33, 125-135. doi:10.1016/j.appdev.2012.03.001 